\title{
Micromechanical analysis of ionic clustering in Nafion perfluorinated membrane
}

\author{
Jiang Yu Li, Sia Nemat-Nasser* \\ Center of Excellence for Advanced Materials, Department of Mechanical and Aerospace Engineering, University of California, San Diego, \\ 9500 Gilman Drive, La Jolla, CA 92093-0416, USA
}

Received 2 August 1999; received in revised form 4 January 2000

\begin{abstract}
The cluster morphology in a water-swollen Nafion perfluorinated membrane is studied using a micromechanics approach. The cluster size is determined from the minimization of the free energy as a function of the equivalent weight of Nafion, the volume fraction of water, and the temperature, taking into account the electrostatic dipole interaction energy, the elastic polymer chain reorganization energy, and the cluster surface energy, leading to results which are in accord with experimental observations. By minimizing the sum of: (1) the electro-elastic interaction energy between an ionic cluster and the fluorocarbon matrix, and (2) the cluster surface energy, it is concluded that the effective cluster shape is spherical in the absence of an electric field, and becoming an oblate spheroid when an electric field is applied. The effect of cluster morphology on the effective electro-elastic moduli and the effective ionic conductivity is then studied by a micromechanical multi-inclusion model. The result seems to describe the available empirical relation when a spherical cluster shape is assumed. It correctly predicts the insulator-to-conductor transition which occurs in Nafion, as the water volume fraction is increased. (c) 2000 Elsevier Science Ltd. All rights reserved.
\end{abstract}

\section{Introduction}

Nafion, a perfluorinated membrane, is a copolymer of tetrafluoroethylene and sulfonyl fluoride vinyl ether, produced by DuPont de Nemours. It has the following chemical structure:

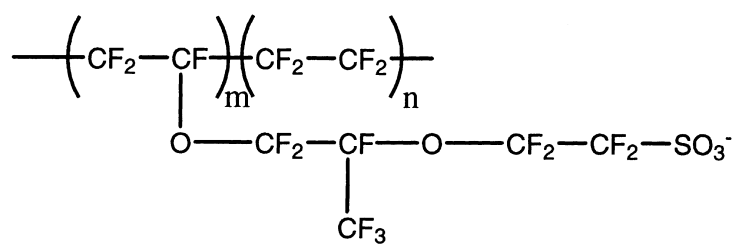

\footnotetext{
${ }^{*}$ Corresponding author. Tel.: +1-858-534-5930; fax: +1-858534-2727.

E-mail address: sia@halebopp.ucsd.edu (S. Nemat-Nasser).
}

This linear fluorocarbon polymer, having some (no more than $10 \mathrm{~mol} \%$ ) pending acid groups, is strongly affected by water content and cations, because it combines two incompatible components, i.e., the hydrophobic fluorocarbon with the hydrophilic ionic phase, and has the phase-separation morphology of discrete hydrophobic and hydrophilic regions. The polytetrafluoroethylene in this material provides the three-dimensionally structured backbone system, having regularly spaced long perfluorovinyl ether pendant side chains which terminate in ionic sulfonate groups. The membrane is permeable to water and cations, while it is impermeable to anions.

It is widely accepted that the ionic groups tend to aggregate to form tightly packed regions 
referred to as clusters (Eisenberg, 1970; Gierke et al., 1981) which are fully interconnected and are readily saturated by water, and have a marked effect on the elastic and transport properties of Nafion. The validity of such a structure has been supported by both theoretical (Eisenberg, 1970; Hsu and Gierke, 1982) and experimental (Gierke et al., 1981; Xue et al., 1989; Lee et al., 1992) observations. Readers are referred to review articles by Mauritz (1988) and Heitner-Wirguin (1996) for more information. It is believed that the ionic clustering is due to the electrostatic interaction among dipoles attached to the fluorocarbon polymer chains (Eisenberg, 1970). This results in an increase in the mean radius of gyration of the polymer chains (Forsman, 1982, 1986) and thus, an increase in the elastic energy associated with their extension. Hsu and Gierke (1982) have developed an elastic theory for the ionic clustering in Nafion, which describes the experimental data well. Their model ignores the electrostatic dipole interaction which can be the driving force for the clustering, and the elastic energy term which is considered is the interaction energy of the ionic cluster and the fluorocarbon matrix, rather than the elastic energy associated with the polymer chain reorganization during clustering. The validity of the assumption that the number of ion exchange sites per cluster is constant during Nafion swelling and cluster growth in Nafion, is also questioned by Gierke et al. (1981). The electrostatic and elastic forces acting on the pendant ionic groups and their neutralizing counterions are shown by Datye et al. (1984) to produce a dipole layer at the surface of an ionic cluster, using a computer simulation. Datye and Taylor (1985) have performed a Monte Carlo simulation to determine the electrostatic energy of an ionic cluster in an ionomer, as a function of the cluster size and temperature. It is found that the energy is not very sensitive to the variation of the cluster shape.

In the present work, we will first determine the cluster size and shape from the minimization of the free energy, taking into account the electrostatic dipole interaction energy, the elastic energy of the polymer chain reorganization during clustering, the surface energy of a cluster, and the electroelastic interaction energy of an ionic cluster and the fluorocarbon polymer matrix. The effect of the cluster morphology on the macroscopic electroelastic and transport properties is then studied, using a micromechanical multi-inclusion model (Nemat-Nasser and Hori, 1993, 1999). Good agreement with experimental data is observed for both cluster morphology and the effective properties of Nafion.

\section{Cluster morphology in Nafion: a micromechanical model}

\subsection{Cluster size in Nafion}

We first consider the cluster size in a waterswollen Nafion membrane, which is assumed to be determined by the electrostatic dipole interaction energy of the ions, the elastic energy of the polymeric chain reorganization, and the surface energy of the cluster. The electro-elastic interaction between a cluster and the fluorocarbon matrix is ignored, since it does not seem to depend on the cluster size, as we will show later on. The electrostatic energy of the clusters arises principally from the interaction between ion pairs. This is modeled as point dipoles in the present calculation. The electrostatic energy, $U_{i j}$, of a pair of dipoles, $\mathbf{m}_{i}$ and $\mathbf{m}_{j}$, is given by (Cheston, 1964)

$$
\begin{aligned}
U_{i j}= & \frac{1}{4 \pi \kappa^{*}}\left\{\frac{\mathbf{m}_{i} \cdot \mathbf{m}_{j}}{\left|\mathbf{r}_{i}-\mathbf{r}_{j}\right|^{3}}\right. \\
& \left.-\frac{3\left[\mathbf{m}_{i} \cdot\left(\mathbf{r}_{i}-\mathbf{r}_{j}\right)\right]\left[\mathbf{m}_{j} \cdot\left(\mathbf{r}_{i}-\mathbf{r}_{j}\right)\right]}{\left|\mathbf{r}_{i}-\mathbf{r}_{j}\right|^{5}}\right\},
\end{aligned}
$$

where $\kappa^{*}$ is the effective dielectric constant of the water-swollen Nafion, and $\mathbf{r}_{i}$ is the position vector of dipole $\mathbf{m}_{i}$. The electrostatic energy of a cluster composed of $N$ dipoles of a common magnitude $m$, which are situated on its surface, then is

$$
\begin{aligned}
U_{\text {ele }}= & \frac{1}{4 \pi \kappa^{*}} \frac{1}{2} \sum_{i=1}^{N} \sum_{j=1}^{N}\left\{\frac{\mathbf{m}_{i} \cdot \mathbf{m}_{j}}{\left|\mathbf{r}_{i}-\mathbf{r}_{j}\right|^{3}}\right. \\
& \left.-\frac{3\left[\mathbf{m}_{i} \cdot\left(\mathbf{r}_{i}-\mathbf{r}_{j}\right)\right]\left[\mathbf{m}_{j} \cdot\left(\mathbf{r}_{i}-\mathbf{r}_{j}\right)\right]}{\left|\mathbf{r}_{i}-\mathbf{r}_{j}\right|^{5}}\right\} .
\end{aligned}
$$


For a given cluster of fixed radius and number of dipoles, the configuration of dipoles on the cluster surface is such that the free energy of the cluster is minimized. In such a situation, the magnitude of $\mathbf{r}_{i}-\mathbf{r}_{j}$ will be proportional to the cluster radius, while its orientation will be independent of the cluster radius. Thus, we can always rewrite Eq. (2) as

$U_{\text {ele }}=-g \frac{N^{2}}{4 \pi \kappa^{*}} \frac{m^{2}}{r_{\mathrm{c}}^{3}}=-\frac{N^{2}}{4 \pi \kappa^{*}} \frac{m^{* 2}}{r_{\mathrm{c}}^{3}}$,

where $g$ is the geometric factor, depending only on the detailed arrangement of the dipoles on the cluster surface, $r_{\mathrm{c}}$ the radius of the cluster, and $m^{*}=\sqrt{g} m$ is the effective dipole moment.

The elastic energy per cluster associated with ionic clustering, is obtained by Datye et al. (1984), using a simple model of rubber elasticity. It is given by

$U_{\text {ela }}=\frac{3 N k T}{4\left\langle h^{2}\right\rangle}\left(d_{0}^{2}+\Delta d^{2}\right)$,

where $k$ is Boltzmann's constant, $T$ the absolute temperature, $\left\langle h^{2}\right\rangle$ the mean square end-to-end chain length, $d_{0}$ the nearest-neighbor distance between dipoles on the cluster, and $\Delta d$ is the average end-to-end separation of the chains belonging to different clusters, given by

$\Delta d=2\left(R-r_{\mathrm{c}}\right)$,

where $R$ is the length of the edge of the cubic volume occupied by the $N$ dipoles before clustering; it is given by

$R^{3}=\frac{N M_{\mathrm{e}}}{\rho^{*} N_{\mathrm{A}}}$,

where $M_{\mathrm{e}}$ is the equivalent weight of Nafion (i.e., the weight in grams of dry polymer per mole of ion exchange sites), $\rho^{*}$ the effective density of the water-swollen Nafion membrane, and $N_{\mathrm{A}}$ is Avogadro's constant. Inserting Eqs. (5) and (6) into Eq. (4), and taking into account that $d_{0} \ll \Delta d$, we obtain

$U_{\text {ela }}=\frac{3 N k T}{\left\langle h^{2}\right\rangle}\left(\sqrt[3]{\frac{N M_{\mathrm{e}}}{\rho^{*} N_{\mathrm{A}}}}-r_{\mathrm{c}}\right)^{2}$.
In deriving Eq. (4), the assumption has been made that half of the chains starting from the pendant ions terminate on the same cluster while the remaining chains terminate on a nearestneighbor cluster (Datye et al., 1984); see Fig. 1.

Introducing the surface energy density $\gamma$, the surface energy of the cluster is simply given by

$U_{\text {sur }}=4 \pi r_{\mathrm{c}}^{2} \gamma$

The surface energy is composed of the hydrophilic energy between the water and the ion pairs, and the hydrophobic energy between the water and the fluorocarbon matrix. A small decrease in the surface energy density $\gamma$ with an increase in the volume fraction of water is expected, since it decreases the influence of the ions.

The energy density of the Nafion membrane due to the ionic clustering is then given by the product of the total energy per cluster due to the ionic clustering (i.e., the sum of the electrostatic dipole interaction energy, the elastic energy of the polymer chain reorganization, and the cluster surface energy) and $n$, the number of clusters per unit volume,

$n=\frac{3\left(c_{\mathrm{w}}+c_{\mathrm{i}}\right)}{4 \pi r_{\mathrm{c}}^{3}}$,

where $c_{\mathrm{w}}$ and $c_{\mathrm{i}}$ are the volume fractions of the water and the ion exchange sites in the membrane,

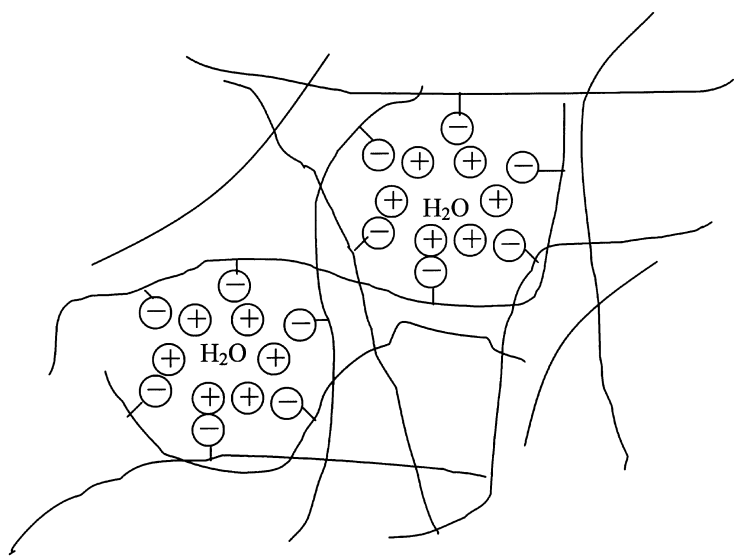

Fig. 1. Schematic illustration of the deformation of polymer chains due to clustering of the pendant ions. 
respectively. The energy density of the membrane is then given by

$$
\begin{aligned}
U= & {\left[-\frac{3 N^{2}}{16 \pi^{2} \kappa^{*}} \frac{m^{* 2}}{r_{\mathrm{c}}^{6}}+\frac{9 N k T}{4 \pi r_{c}^{3}\left\langle h^{2}\right\rangle}\right.} \\
& \left.\times\left(\sqrt[3]{\frac{N M_{\mathrm{e}}}{\rho^{*} N_{\mathrm{A}}}}-r_{\mathrm{c}}\right)^{2}+\frac{3 \gamma}{r_{\mathrm{c}}}\right]\left(c_{\mathrm{w}}+c_{\mathrm{i}}\right) .
\end{aligned}
$$

The number of dipoles per cluster, $N$, can be obtained directly from the density and the equivalent weight of the membrane (Gierke et al., 1981),

$N=\frac{N_{\mathrm{A}} \rho_{\mathrm{d}}}{M_{\mathrm{e}}(1+\Delta V)} L^{3}$,

where $\rho_{\mathrm{d}}$ is the density of the dry membrane, $\Delta V$ the fractional volume change of the membrane due to water absorption, and

$L=\sqrt[3]{\frac{4 \pi}{3\left(c_{\mathrm{w}}+c_{\mathrm{i}}\right)}} r_{\mathrm{c}}$

is the mean distance between clusters. Eq. (10) can then be rewritten as

$$
\begin{aligned}
U= & \left\{-\frac{1}{3 \kappa^{*}}\left[\frac{N_{\mathrm{A}} \rho_{\mathrm{d}} m^{*}}{M_{\mathrm{e}}(1+\Delta V)\left(c_{\mathrm{w}}+c_{\mathrm{i}}\right)}\right]^{2}\right. \\
& +\frac{3 k T}{\left\langle h^{2}\right\rangle} \frac{N_{\mathrm{A}} \rho_{\mathrm{d}}}{M_{\mathrm{e}}(1+\Delta V)\left(c_{\mathrm{w}}+c_{\mathrm{i}}\right)} \\
& \left.\times\left[\sqrt[3]{\frac{4 \pi \rho_{\mathrm{d}}}{3 \rho^{*}(1+\Delta V)\left(c_{\mathrm{w}}+c_{\mathrm{i}}\right)}}-1\right]^{2} r_{\mathrm{c}}^{2}+\frac{3 \gamma}{r_{\mathrm{c}}}\right\} \\
& \times\left(c_{\mathrm{w}}+c_{\mathrm{i}}\right) .
\end{aligned}
$$

Differentiating Eq. (10a) with respect to $r_{\mathrm{c}}$, and setting the result equal to zero, we obtain

$$
\begin{aligned}
& \frac{6 k T}{\left\langle h^{2}\right\rangle} \frac{N_{\mathrm{A}} \rho_{\mathrm{d}}}{M_{\mathrm{e}}(1+\Delta V)\left(c_{\mathrm{w}}+c_{\mathrm{i}}\right)} \\
& \quad \times\left[\sqrt[3]{\frac{4 \pi \rho_{\mathrm{d}}}{3 \rho^{*}(1+\Delta V)\left(c_{\mathrm{w}}+c_{\mathrm{i}}\right)}}-1\right]^{2} r_{\mathrm{c}}-\frac{3 \gamma}{r_{\mathrm{c}}^{2}}=0,
\end{aligned}
$$

so that

$$
\begin{aligned}
r_{\mathrm{c}}^{3}= & \frac{\gamma\left\langle h^{2}\right\rangle M_{\mathrm{e}}}{2 N_{\mathrm{A}} k T} \frac{\left(\Delta V+\Delta V^{\prime}\right)}{\rho_{\mathrm{d}}} \\
& \times\left[1-\sqrt[3]{\frac{4 \pi \rho_{\mathrm{d}}}{3 \rho^{*}\left(\Delta V+\Delta V^{\prime}\right)}}\right]^{-2},
\end{aligned}
$$

where

$c_{\mathrm{w}}=\frac{\Delta V}{1+\Delta V} \quad$ and $\quad c_{\mathrm{i}}=\frac{\Delta V^{\prime}}{1+\Delta V}$

have been used; here

$\Delta V^{\prime}=\frac{N_{\mathrm{A}} V_{\mathrm{i}}}{M_{\mathrm{e}} / \rho_{\mathrm{d}}}$

is the volume fraction of the ion exchange sites in the dry membrane, with $V_{\mathrm{i}}$ being the volume of a single ion exchange site, and

$\rho^{*}=\frac{\rho_{\mathrm{d}}+\Delta V \rho_{\mathrm{w}}}{1+\Delta V}$

is the effective density. Assuming $\left\langle h^{2}\right\rangle=\beta M_{\mathrm{e}}$ (Forsman, 1986), Eq. (13) suggests that a plot of $r_{\mathrm{c}}^{3}$ versus

$\Phi=\frac{M_{\mathrm{e}}^{2}\left(\Delta V+\Delta V^{\prime}\right)}{\rho_{\mathrm{d}}}\left[1-\sqrt[3]{\frac{4 \pi \rho_{\mathrm{d}}}{3 \rho^{*}\left(\Delta V+\Delta V^{\prime}\right)}}\right]^{-2}$

should be a straight line crossing the origin for all membranes of different equivalent weight, different cations, or water intake, with the slope given by $\gamma \beta / 2 N_{\mathrm{A}} k T$. Typical value for $\Phi / M_{\mathrm{e}}^{2}$ is 0.19 for $\Delta V=0.443$.

To verify the theory, we have compared the model result with the experimentally determined cluster diameters $\left(d_{\mathrm{c}}\right)$ of Nafions with different amounts of water contents, different equivalent weights, and different cation forms, obtained by Gierke et al. (1981), using small-angle X-ray scanning. Their results are summarized in Tables $1-3$, and the functional dependence of $d_{\mathrm{c}}^{3}$ on $\Phi$ is plotted in Figs. 2-4, for Nafion with different water absorption, different equivalent weights, and different cation forms, respectively. To calculate $\Phi$, we have used $V_{\mathrm{i}}=68 \times 10^{-24} \mathrm{~cm}^{3}$ (Gierke et al., 1981). It is found that Eq. (13) which predicts a linear relation between $d_{\mathrm{c}}^{3}$ and $\Phi$, describes the experimental data very well. The straight lines 
Table 1

Cluster size of 1200 equivalent weight Nafions with different water content

\begin{tabular}{lcccccccc}
\hline Cation & $\mathrm{H}^{+}$ & $\mathrm{Li}^{+}$ & $\mathrm{Li}^{+}$ & $\mathrm{H}^{+}$ & $\mathrm{Li}^{+}$ & $\mathrm{Li}^{+}$ & $\mathrm{H}^{+}$ & $\mathrm{Li}^{+}$ \\
\hline Dry density $\left(\mathrm{g} / \mathrm{cm}^{3}\right)$ & 2.075 & 2.078 & 2.078 & 2.075 & 2.078 & 2.078 & 2.075 & 2.078 \\
Volume gain $(\%)$ & 41 & 39.1 & 32.3 & 27.2 & 25.3 & 18.2 & 13.3 & 11.4 \\
Cluster diameter $(\mathrm{nm})$ & 4.08 & 3.94 & 3.70 & 3.38 & 3.27 & 2.97 & 2.52 & 2.52 \\
\hline
\end{tabular}

Table 2

Cluster size of Nafions in $\mathrm{Na}^{+}$form with different equivalent weights

\begin{tabular}{lcccccc}
\hline Equivalent weight & 944 & 971 & 1100 & 1200 & 1600 & 1790 \\
\hline Dry density $\left(\mathrm{g} / \mathrm{cm}^{3}\right)$ & 2.088 & 2.093 & 2.103 & 2.113 & 2.135 & 2.144 \\
Volume gain $(\%)$ & 87.4 & 78.2 & 49.8 & 37.5 & 17.2 & 13.3 \\
Cluster diameter $(\mathrm{nm})$ & 5.09 & 4.97 & 4.31 & 3.88 & 3.03 & 2.74 \\
\hline
\end{tabular}

Table 3

Cluster size of 1200 equivalent weight Nafions with different cations

\begin{tabular}{lcccccc}
\hline Cation & $\mathrm{H}^{+}$ & $\mathrm{Li}^{+}$ & $\mathrm{Na}^{+}$ & $\mathrm{K}^{+}$ & $\mathrm{Rb}^{+}$ & $\mathrm{Cs}^{+}$ \\
\hline Dry density $\left(\mathrm{g} / \mathrm{cm}^{3}\right)$ & 2.075 & 2.078 & 2.113 & 2.141 & 2.221 & 2.304 \\
Volume gain $(\%)$ & 69.7 & 61.7 & 44.3 & 18.7 & 17.9 & 13.6 \\
Cluster diameter $(\mathrm{nm})$ & 4.74 & 4.49 & 4.21 & 3.45 & 3.56 & 3.50 \\
\hline
\end{tabular}

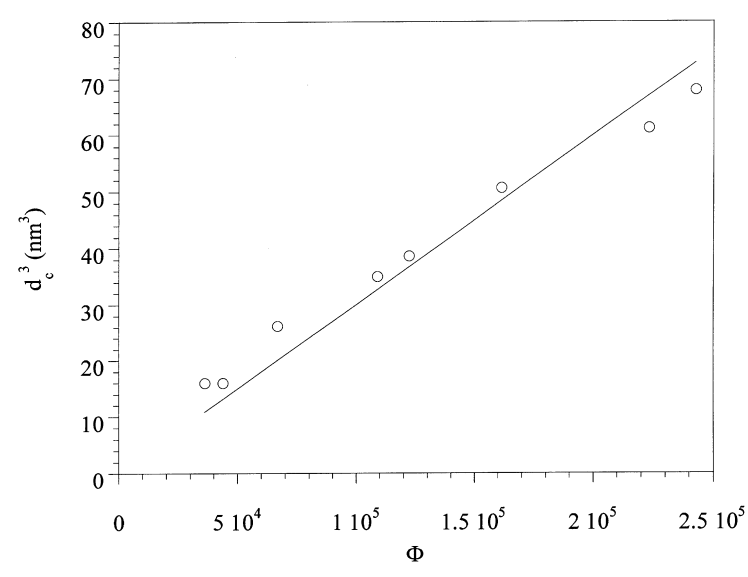

Fig. 2. Cluster size in Nafion membranes with different water content.

cross the origin for Nafions with different water contents or equivalent weights, as predicted, but not for Nafions with different cations. This is probably due to the fact that different cations have different volumes of ion exchange sites, $V_{\mathrm{i}}$, and in some cases the differences are quite large, e.g., in

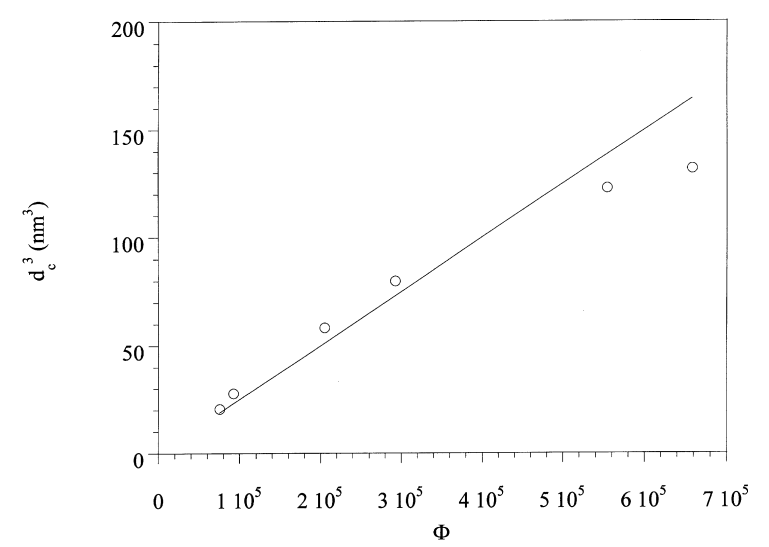

Fig. 3. Cluster size in Nafion membranes with different equivalent weights.

the case of $\mathrm{Li}^{+}$versus $\mathrm{Cs}^{+}$. It is also observed that the slope decreases slightly with an increase in $\Phi$, which is believed to be due to the decrease of the surface energy density $\gamma$, with an increase in the volume fraction of water, decreasing the influence of the ions. 


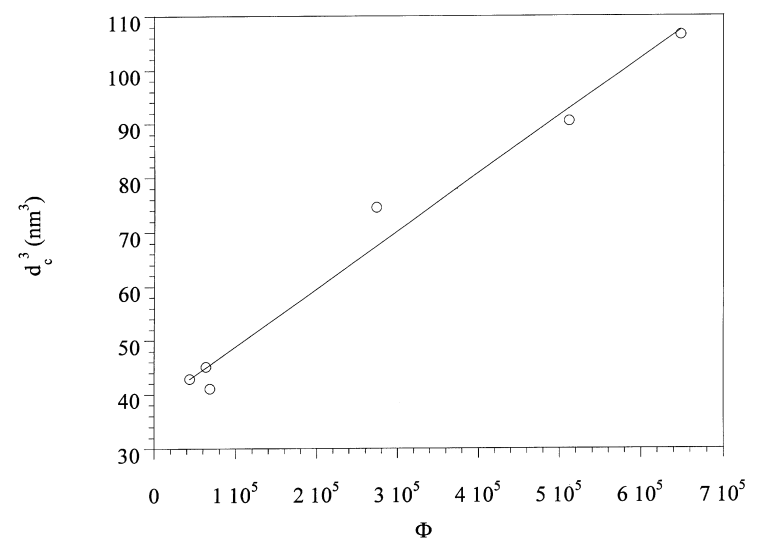

Fig. 4. Cluster size in Nafion membranes with different cation forms.

\subsection{Cluster shape in Nafion}

Next, let us consider the cluster shape in Nafion, which is assumed to be determined by the electro-elastic interaction energy between the ionic clusters and the fluorocarbon matrix, and the surface energy of the cluster. It is assumed that on the average, the electrostatic dipole interaction energy and the elastic energy of the polymer chain reorganization do not depend on the cluster shape. In other words, these energies can be represented by the energy of an effective spherical cluster, discussed in the preceding subsection. The insensitivity of the electrostatic dipole energy to the variation of the cluster geometry has been demonstrated by Datye and Taylor (1985). In order to treat the elastic and electric variables on equal footing, we introduce the following notation originally proposed by Barnett and Lothe (1975) for piezoelectricity: both lower-case and uppercase subscripts are used, with the upper-case subscript ranging from 1 to 4 , where 1 to 3 are for the elastic variables and 4 is for the electric variables; the repeated upper-case subscripts are summed from 1 to 4 . Using this notation, the electro-elastic variables can be written as

$$
\begin{aligned}
& \Sigma_{i J}=\left[\begin{array}{l}
\sigma_{i j} \\
D_{i}
\end{array}\right], \quad Z_{K l}=\left[\begin{array}{l}
\varepsilon_{k l} \\
E_{l}
\end{array}\right], \\
& G_{i J K l}=\left[\begin{array}{cc}
C_{i j k l} & 0 \\
0 & \kappa_{i l}
\end{array}\right],
\end{aligned}
$$

where $\sigma_{i j}$ and $\Sigma_{k l}$ are the stress and strain tensors, respectively; $D_{i}$ and $E_{l}$ are the electric displacement and the electric field, respectively; $C_{i j k l}$ and $\kappa_{i l}$ are the elastic stiffness tensor and the dielectric constants of the polymer in the absence of any clusters, respectively.

We consider the interaction between the ionic clusters and the fluorocarbon matrix arising from an eigenfield in the cluster. Adopting Mura's (1987) terminology, we refer to a cluster as an inclusion, with eigenfield

$Z_{K l}^{\mathrm{T}}=\left[\begin{array}{c}\varepsilon_{k l}^{\mathrm{T}} \\ E_{l}^{\mathrm{T}}\end{array}\right]$,

so that the constitutive equation for the cluster can be written as

$\Sigma_{i J}=G_{i J K l}\left(Z_{K l}-Z_{K l}^{\mathrm{T}}\right)$,

where $\varepsilon_{k l}^{\mathrm{T}}$ and $E_{l}^{\mathrm{T}}$ are the eigenfield in the cluster, necessary for homogenization; see Nemat-Nasser and Hori $(1993,1999)$. Note that, with the introduction of an eigenfield within the inclusion, a homogeneous medium with the matrix properties, is used to obtain the actual fields within the original heterogeneous material. The interaction energy between the cluster and the matrix due to the presence of an eigenfield is then given by

$$
\begin{aligned}
U_{\text {int }} & =-\frac{1}{2} \Sigma_{i J} Z_{J i}^{\mathrm{T}} \\
& =-\frac{1}{2} G_{i J K l} Z_{J i}^{\mathrm{T}}\left(S_{K l M n}-I_{K l M n}\right) Z_{M n}^{\mathrm{T}},
\end{aligned}
$$

where $Z_{K l}=S_{K l M n} Z_{M n}^{\mathrm{T}}$ is the resulting field when the transformation field is introduced in the inclusion in the presence of the surrounding elastic matrix (Eshelby, 1957),

$I_{K l M n}=\left[\begin{array}{cc}I_{k l m n}^{4} & 0 \\ 0 & i_{\ln }^{2}\end{array}\right]$,

with $I_{k l m n}^{4}$ and $i_{\ln }^{2}$ being the fourth- and secondorder unit tensors, and $S_{K I M n}$ is the Eshelby tensor which is a function of the electro-elastic moduli of the matrix and the aspect ratios of the ellipsoidal cluster. The closed-form expressions for Eshelby tensor for a spheroid in an isotropic medium are tabulated in Mura (1987) for fourth-order tensors, 
and in Taya and Arsenault (1989) for second-order tensors.

Eq. (16a) can be used to determine the electroelastic interaction energy between an ionic cluster and the fluorocarbon matrix, as a function of the cluster shape which is defined by its aspect ratios. It is found that when the eigenfield of an inclusion embedded in an isotropic medium is such that only $\varepsilon_{11}^{\mathrm{T}}=\varepsilon_{22}^{\mathrm{T}}=\varepsilon_{33}^{\mathrm{T}}=\varepsilon_{s}$, and $E_{3}^{\mathrm{T}}=E_{s}$ are non-zero, the interaction energy is given by

$U_{\mathrm{int}}=\frac{2 Y \varepsilon_{s}^{2}}{1-v}+\kappa E_{s}^{2}\left(1-S_{4343}\right)$

where $Y, v$, and $\kappa$ are Young's modulus, Poisson ratio, and dielectric constant, respectively; $S_{4343}$ is the component of the dielectric Eshelby tensor, which is a function of inclusion aspect ratio $\beta$. Eq. (16b) suggests that the interaction energy is independent of the cluster aspect ratio, $\beta$, when $E_{s}$ is zero. This is because for an isotropic medium, we have $S_{i i j j}=(1+v) /(1-v)$ for all aspect ratios. In such a situation, a spherical cluster produces the minimum surface energy. However, when $E_{s}$ is also non-zero (besides the volumetric eigenstrain), it is found that the interaction energy is minimum for penny-shaped clusters, and maximum for cylindrical clusters. The actual aspect ratio of a cluster therefore will depend on a balance between the surface energy and the interaction energy, the determination of which requires a knowledge of both the surface energy density and the magnitude of the eigenfield. An illustration is given in Fig. 5, for spheroids of revolution with aspect ratio $\beta$, defined as

$\beta=\frac{l_{3}}{l_{1}}=\frac{l_{3}}{l_{2}}$,

where $l_{1}, l_{2}$, and $l_{3}$ are the principal axes of the ellipsoidal cluster. In the calculation, the matrix material constants that were used are: dielectric constant $\kappa=4 \kappa_{0}$, Young's modulus $Y=275 \mathrm{MPa}$, and the Poisson ratio, $v=0.487$; the eigenfield are $\varepsilon_{s}=10^{-3}$ and $P_{3}^{s}=\kappa E_{s}=10^{-4} \mathrm{C} / \mathrm{m}^{2}$, where $P_{3}^{s}$ is the spontaneous polarization in the cluster which may be induced by ion redistribution. For comparison, the spontaneous polarization in a ferroelectric $\mathrm{BaTiO}_{3}$ crystal is in the order of $10^{-2} \mathrm{C} / \mathrm{m}^{2}$.

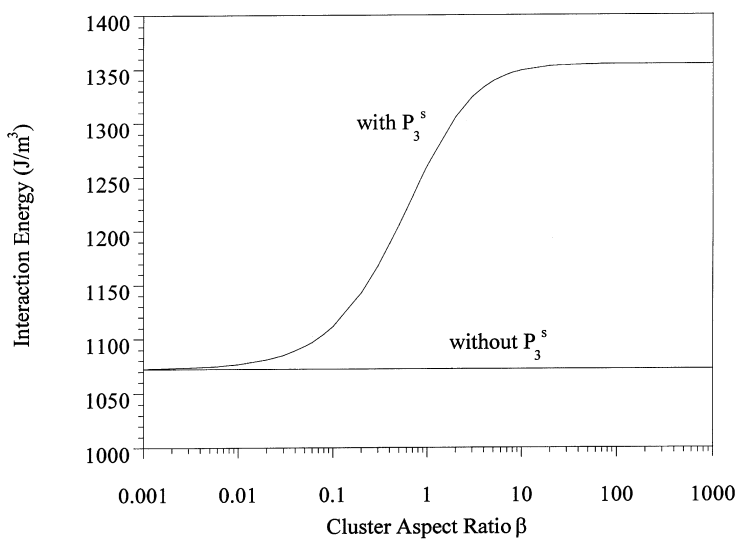

Fig. 5. Electro-elastic interaction energy between ionic cluster and fluorocarbon matrix.

It is hoped that the analysis here provides some insight into the response of ionic polymer metal composites (IPMC) under an applied voltage (Shahinpoor, 1992; Oguro et al., 1993; NematNasser and $\mathrm{Li}, 1999)$ which induces an effective dipole in the clusters, due to the redistribution of ions. Such an induced dipole, according to our calculations, will change the cluster shape, and may cause the bending of the IPMC under an electric field. This could be one possible mechanism defining the instantaneous response of an IPMC to a suddenly applied electric field.

\section{The effective properties of Nafion: a homogeni- zation approach}

\subsection{The effective electro-elastic moduli}

With the phase-separation morphology of the discrete hydrophobic fluorocarbon matrix and the hydrophilic ionic clusters, we can regard a waterswollen Nafion as a two-phase composite material, with the constitutive equation given by

$\left\langle\Sigma_{i J}\right\rangle=\bar{G}_{i J K l}\left\langle Z_{k L}\right\rangle$

where $\langle\cdot\rangle$ is used to denote the volume average, and $\bar{G}_{i J K l}$ is the effective electro-elastic modulus tensor of Nafion. This effective modulus tensor can be rigorously expressed as 
$\bar{G}_{i J K l}=\sum_{r=1}^{n} c_{r} G_{i J M n}^{(r)} A_{M n K l}^{(r)}$,

where $c_{r}$ and $A_{M n K l}^{(r)}$ are the volume fraction and the concentration tensor of phase $r$, respectively, with $A_{M n K l}^{(r)}$ defined by $\left\langle Z_{M n}^{(r)}\right\rangle=A_{M n K l}^{(r)}\left\langle Z_{K l}\right\rangle$. In the following discussion, we reserve the superscript 1 for the matrix, and the superscripts from 2 to $n$ for the inclusions (i.e., clusters). In order to determine the effective moduli of this composite, we need to determine the average field in a typical phase $r$. Various micromechanics models have been proposed for estimating the effective moduli of heterogeneous materials. These include the dilute model, the self-consistent approach (Kröner, 1958; Hill, 1965; Budiansky, 1965), the Mori-Tanaka approach (Mori and Tanaka, 1973; Benveniste, 1987), and the multi-inclusion model (NematNasser and Hori, 1993, 1999). Here we use the multi-inclusion model which includes the MoriTanaka approach and the self-consistent approach as special cases. In this model, it is assumed that the average fields in the inclusions are the same as the fields in a multi-inclusion embedded in an infinite homogeneous medium with electroelastic moduli $G_{i J K l}$, so that the effective overall moduli are given by

$$
\begin{aligned}
\bar{G}_{i J K l}= & G_{i J M n}\left[I_{M n A b}+\left(S_{M n C d}-I_{M n C d}\right) \Lambda_{C d A b}\right] \\
& \times\left(I_{K l A b}+S_{K l E f} \Lambda_{E f A b}\right)^{-1}
\end{aligned}
$$

where the superscript -1 is used to denote the inverse, and

$\Lambda_{K l M n}=\sum_{r=1}^{n} c_{r}\left(\Lambda_{M n K l}^{(r)}-S_{M n K l}\right)^{-1}$,

with $\Lambda_{K l M n}^{(r)}=\left(G_{i J K l}-G_{i J K l}^{(r)}\right)^{-1} G_{i J M n}$. It is seen from Eqs. (19) and (20) that the effective moduli of the multi-inclusion model depend on the choice of the moduli of the infinite medium, $G_{i J M n}$. By assigning $G_{i J M n}^{(1)}$, the moduli of the matrix, to be those of the infinite medium, the results of the multi-inclusion model coincide with those obtained by the MoriTanaka approach which assumes that the average field in a phase is equal to the field in a single inclusion embedded in the infinite matrix, and sub- jected at infinity to the yet unknown field $\left\langle Z_{M n}^{(1)}\right\rangle$. The concentration tensor is then given by

$A_{M n K l}^{(r) \mathrm{MT}}=A_{M n J i}^{(r) \mathrm{dil}}\left(\sum_{r=1}^{n} c_{r} A_{K l J i}^{(r) \mathrm{dil}}\right)^{-1}$,

where the concentration tensor $A_{M n J i}^{(r) \text { dil }}$ is for the dilute model; it is determined using Eshelby's (1959) equivalent inclusion concept as follows (see Nemat-Nasser and Hori, 1993, 1999):

$A_{M n K l}^{(r) \text { dil }}=\left[I_{K l M n}+S_{K l J i}^{(r)}\left(G_{a B J i}^{(1)}\right)^{-1}\left(G_{a B M n}^{(r)}-G_{a B M n}^{(1)}\right)\right]^{-1}$.

Eqs. (19)-(22) can then be used to study the effects of the water volume fraction and the cluster shape on the effective moduli of the water-swollen Nafion.

The theoretical model can be compared with the available experimental data. The tensile modulus of the water-swollen Nafion has been reported (Grot et al., 1972; Hsu and Gierke, 1982) to obey the following empirical relation:

$Y(f)=Y_{0} \operatorname{Exp}\left[-\alpha\left(f+\frac{1200-M_{\mathrm{e}}}{20}\right)\right]$

with $Y_{0}=275 \mathrm{MPa}$, and $\alpha=0.0294$, and where $f=100 \Delta m$ is grams of water per 100 grams of dry membrane, $\Delta m$ being the fractional weight change of the Nafion due to swelling. The fractional volume change of the Nafion due to swelling can be written as

$\Delta V=\frac{\rho_{\mathrm{d}} \Delta m}{\rho_{\mathrm{w}}}$,

and the water volume fraction $c_{\mathrm{w}}$ in the Nafion can be expressed as

$c_{\mathrm{w}}=\frac{\Delta V}{1+\Delta V}=\frac{\rho_{\mathrm{d}} f}{100 \rho_{\mathrm{w}}+\rho_{\mathrm{d}} f}$,

so that

$Y\left(c_{\mathrm{w}}\right)=Y_{0} \operatorname{Exp}\left[-\alpha\left(\frac{100 c_{\mathrm{w}} \rho_{\mathrm{w}}}{\left(1-c_{\mathrm{w}}\right) \rho_{\mathrm{d}}}+\frac{1200-M_{\mathrm{e}}}{20}\right)\right]$.

Eq. (26) gives an empirical relation for the tensile modulus of Nafion as a function of its water vol- 
ume fraction. We use Eq. (26) to represent the experimental data, and compare the results with those of the multi-inclusion and the Mori-Tanaka models, as presented in Figs. 6-11 for Nafion with indicated equivalent weight. In the micromechanical calculation, three cluster aspect ratios, 0.1, 1, and 10, are used. The Young modulus of the dry Nafion is determined from Eq. (23) for different equivalent weights, and the Poisson ratio is assumed to be 0.487 for all equivalent weights. The bulk modulus of water is taken to be $2.55 \mathrm{Gpa}$, and its shear modulus is assumed to be zero. It is

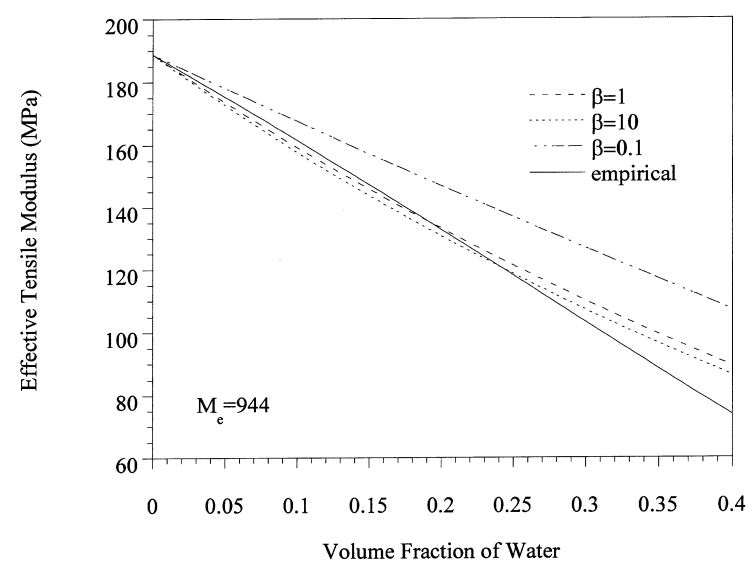

Fig. 6. Effective tensile modulus of 944 equivalent weight Nafion membrane.

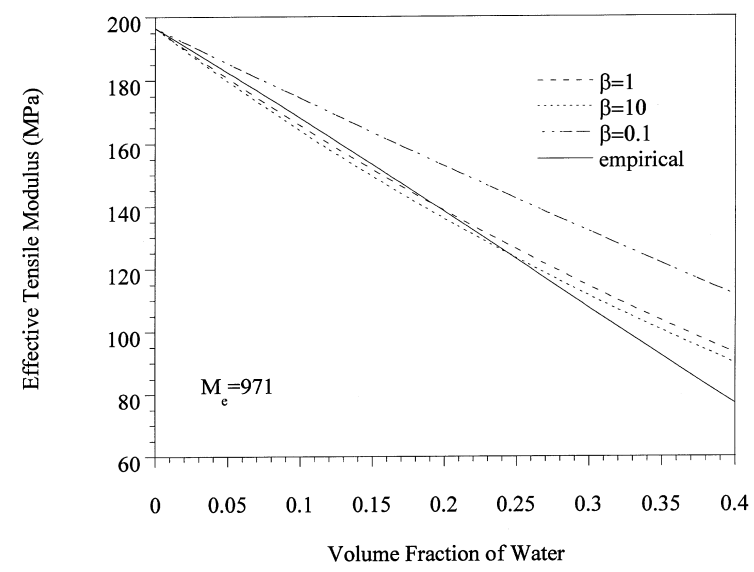

Fig. 7. Effective tensile modulus of 971 equivalent weight Nafion membrane.

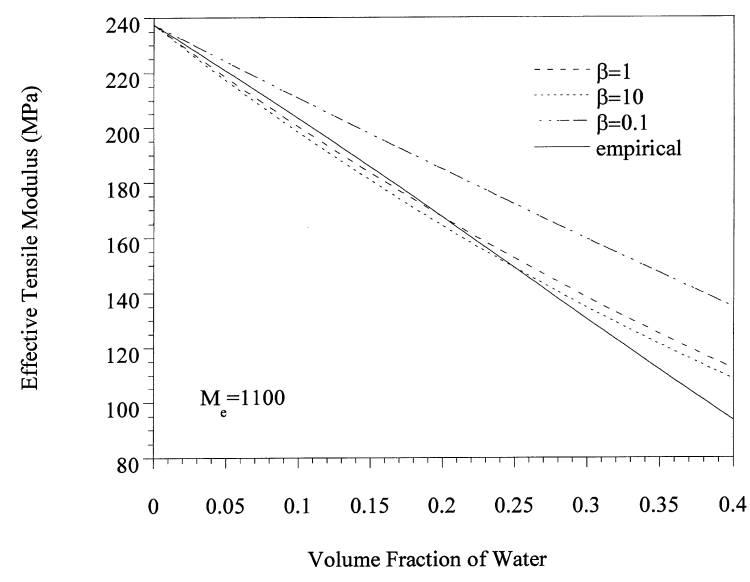

Fig. 8. Effective tensile modulus of 1100 equivalent weight Nafion membrane.

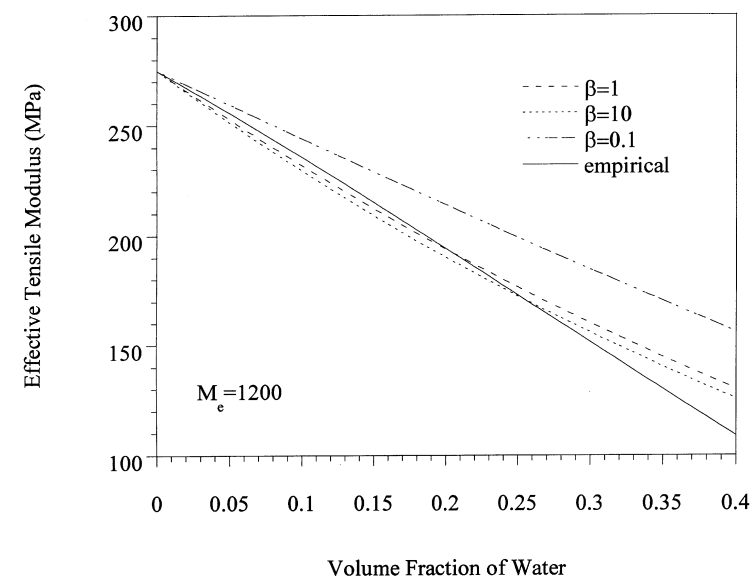

Fig. 9. Effective tensile modulus of 1200 equivalent weight Nafion membrane.

observed that for Nafions with different equivalent weights, the calculation using spherical cluster shape, best fits the empirical relations. This provides another piece of evidence to show that, without an applied electric field, the clusters in Nafion are more or less spherical.

\subsection{The effective conductivity}

The effective conductivity, $\bar{\lambda}_{i j}$, can be defined in a similar manner as the effective electro-elastic moduli, 


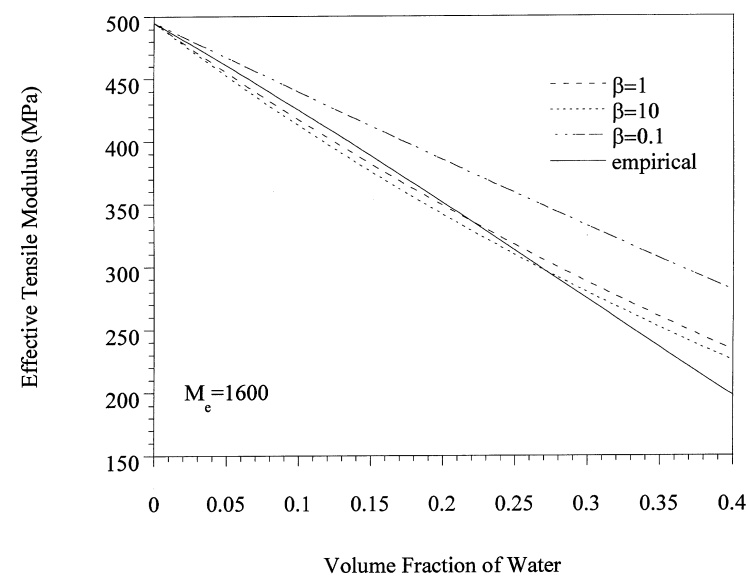

Fig. 10. Effective tensile modulus of 1600 equivalent weight Nafion membrane.

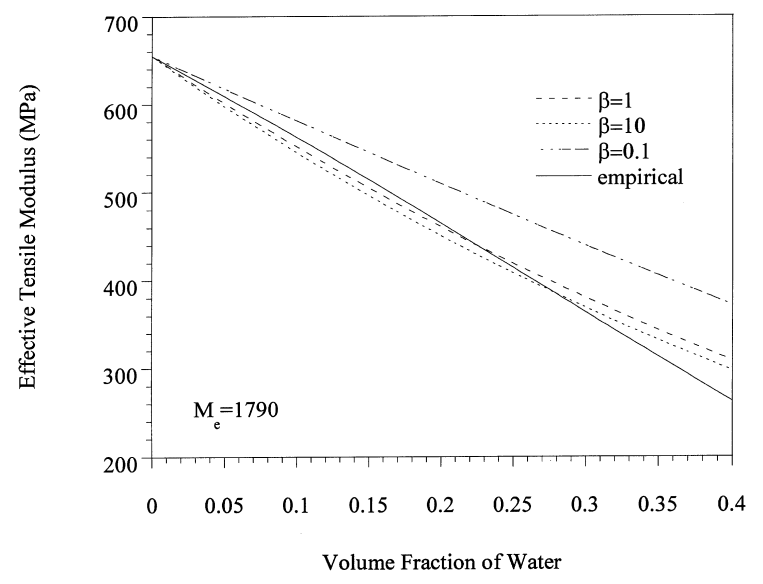

Fig. 11. Effective tensile modulus of 1790 equivalent weight Nafion membrane.

$\left\langle J_{\mathrm{i}}\right\rangle=\bar{\lambda}_{i j}\left\langle\frac{\partial C}{\partial x_{j}}\right\rangle$

where $J_{\mathrm{i}}$ is the ionic flux, and $C$ is the ion density. It can be expressed rigorously as

$\bar{\lambda}_{i j}=\sum_{r=1}^{n} c_{r} \lambda_{i k}^{(r)} a_{k j}^{(r)}$

with the concentration factor $a_{i j}^{(r)}$ defined by $\left\langle\frac{\partial C^{(r)}}{\partial x_{i}}\right\rangle=a_{i j}^{(r)}\left\langle\frac{\partial C}{\partial x_{j}}\right\rangle$

To estimate the effective conductivity of Nafion, we use the multi-inclusion model, as given by Eqs. (19) and (20) for the electro-elastic moduli, though we need to be careful in choosing the modulus for the infinite medium. Because of the great difference in the ionic conductivity between water and the fluorocarbon matrix, assigning the matrix modulus to the infintie medium, which results in the Mori-Tanaka model, does not produce a good estimate of the effective conductivity of the waterswollen Nafion. To predict the effective conductivity of the water-swollen Nafion, we set the conductivity of the infinite medium to be the yet unknown overall effective conductivity of the Nafion, as is done in the self-consistent model. This gives

$a_{k l}^{(r) s c}=\left[i_{l k}^{2}+s_{l m}^{(r)} \bar{\lambda}_{m n}^{-1}\left(\lambda_{n k}^{(r)}-\bar{\lambda}_{n k}\right)\right]^{-1}$,

where $s_{l m}^{(r)}$ is Eshelby tensor (second-order) for phase $r$. In the self-consistent model, $s_{l m}^{(r)}$ is a function of the effective conductivity of Nafion and the aspect ratios of the cluster. The closedform expression of this tensor for a spheroid embedded in an isotropic medium is tabulated in Taya and Arsenault (1989). Eqs. (28) and (29) can be used to study the effects of the water volume fraction and the cluster shape on the effective conductivity. For a spherical cluster, the effective conductivity is determined as

$$
\begin{aligned}
\bar{\lambda}= & \frac{1}{4}\left\{\left(2-3 c_{\mathrm{w}}\right) \lambda_{\mathrm{p}}+\left(3 c_{\mathrm{w}}-1\right) \lambda_{\mathrm{w}}\right. \\
& \left.+\sqrt{8 \lambda_{\mathrm{p}} \lambda_{\mathrm{w}}+\left[\left(3 c_{\mathrm{w}}-2\right) \lambda_{\mathrm{p}}+\left(1-3 c_{\mathrm{w}}\right) \lambda_{\mathrm{w}}\right]^{2}}\right\},
\end{aligned}
$$

where $\lambda_{\mathrm{w}}$ and $\lambda_{\mathrm{p}}$ are the conductivity of water and dry Nafion, respectively.

An empirical relation for the effective conductivity has been reported for Nafion by Hsu et al. (1980). This experimental result exhibits an insulator-to-conductor transition. The empirical relation is 


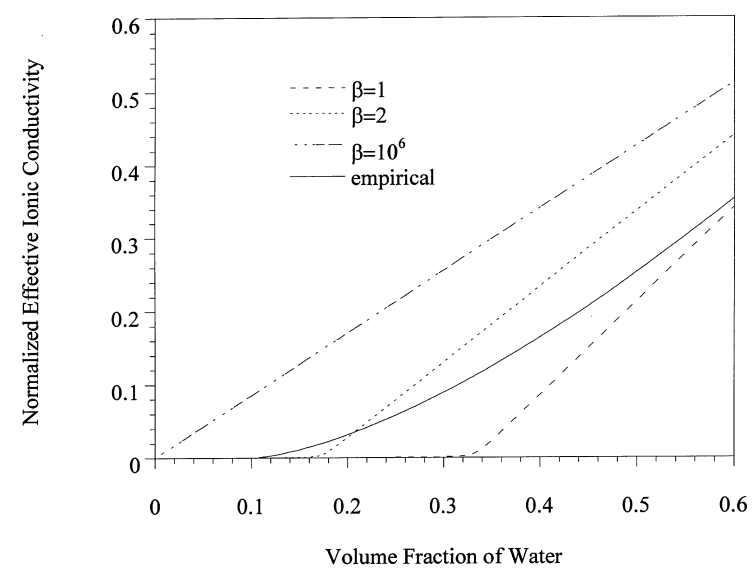

Fig. 12. Effective conductivity of Nafion membrane.

$\bar{\lambda}=\lambda_{0}\left(c_{\mathrm{w}}-c_{0}\right)^{\eta}$

with $c_{0}=0.08-0.1$, and $\eta=1.53 \pm 0.1$. We have compared the results of this empirical relation with those predicted by the multi-inclusion model in Fig. 12. It is seen that the self-consistent assumption describes the empirical relation well, and predicts the phenomenon of the insulator-to-conductor transition.

\section{Conclusion}

We have studied the cluster morphology in the water-swollen Nafion perfluorinated membrane by minimization of the free energy. In this manner, the cluster size is determined by assuming the free energy is a function of the equivalent weight of Nafion, the volume fraction of the water, and the temperature. The results are in good accord with the experimental data. The cluster shape is predicted to favor a sphere in the absence of an electric field, and an oblate spheroid when an electric field is applied. The effect of the cluster morphology on the effective electro-elastic moduli and on the effective ionic conductivity is studied using a micromechanical multi-inclusion model. The results describe the available empirical relations well for the spherical cluster shape. They correctly predict the insulator-to-conductor tran- sition which occurs in Nafion with an increased water volume fraction.

\section{Acknowledgements}

The work reported here has been supported in part by Defense Advanced Projects Agency (DARPA, Dr. Steve Wax, Program Manager) and in part by the UCSD endowed John Dove Isaacs Chair in Natural Philosophy.

\section{References}

Barnett, D.M., Lothe, J., 1975. Dislocations and line charges in anisotropic piezoelectric insulators. Phys. Status Sol. (B) 67, 105-111.

Benveniste, Y., 1987. A new approach to the application of Mori-Tanaka's theory in composite materials. Mech. Mater. 6, 147-157.

Budiansky, B., 1965. On the elastic moduli of some heterogeneous materials. J. Mech. Phys. Solids 3, 223-227.

Cheston, W.B., 1964. Elementary Theory of Electric and Magnetic Fields. Wiley, New York.

Datye, V.K., Taylor, P.L., Hopfinger, A.J., 1984. Simple model for clustering and ionic transport in ionomer membranes. Macromolecules 17, 1704-1708.

Datye, V.K., Taylor, P.L., 1985. Electrostatic constributions to the free energy of clustering of an ionomer. Macromolecules $18,1479-1482$.

Eisenberg, A., 1970. Clustering of ions in organic polymers, a theoretical approach. Macromolecules 3, 147-154.

Eshelby, J.D., 1957. The determination of the elastic field of an ellipsoidal inclusion, and related problems. Proc. R. Soc. London. Ser. A. 241, 376-396.

Forsman, W.A., 1982. Effect of segment-segment association on chain dimensions. Macromolecules 15, 1032-1040.

Forsman, W.A., 1986. Statistical mechanics of ion-pair association in ionomers. In: Proceedings of the NATO Advanced Workshop on Structure and Properties of Ionomers, pp. 3950 .

Gierke, T.D., Munn, G.E., Wilson, F.G., 1981. The morphology in nafion perfluorinated membrane products, as determined by wide- and small-angle X-ray studies. J. Polym. Sci., Polym. Phys. Ed. 19, 1687-1704.

Grot, W.G.F., Munn, C.E., Walmsley, P.N., 1972. In: 141st Meeting of the Electrochemical Society, Houston, Texas, May, 1972.

Heitner-Wirguin, C., 1996. Recent advances in perfluorinated ionmer membranes: structure, properties and applications. J. Membrane Sci. 120, 1-33.

Hill, R., 1965. A self-consistent mechanics of composites materials. J. Mech. Phys. Solids 13, 213-222. 
Hsu, W.Y., Barkley, J.R., Meakin, P., 1980. Ion percolation and insulator-to-conductor transition in nafion perfluorosulfonic acid membranes. Macromolecules 13, 198-200.

Hsu, W.Y., Gierke, T.D., 1982. Elastic theory for ionic clustering in perfluorinated ionomers. Macromolecules 15, 101-105.

Kröner, E., 1958. Berechnung der elastischen Konstanten des Vielkristalls aus den Konstanten des Einkristalls. Z. Phys. 151, 504-518.

Lee, E.M., Thomas, R.K., Burgess, A.N., Barnes, D.Y., Soper, A.K., Rennil, A.R., 1992. Local and long range structure of water in perfluorinated ionomer membrane. Macromolecules 25, 3106-3109.

Mauritz, K.A., 1988. Review and critical analysis of theories of aggregation in ionomers. J. Macromol. Sci., Rev. Macromol. Chem. Phys. 28 (1), 65-98.

Mori, T., Tanaka, K., 1973. Average stress in matrix and average elastic energy of materials with misfitting inclusions. Acta Mettall. 21, 571-574.
Mura, T., 1987. Micromechanics of Defects in Solids, second ed. Martinus Nijhoff, Dordrecht.

Nemat-Nasser, S., Hori, M., 1993. Micromechanics: Overall Properties of Heterogeneous Materials, second ed. Elsevier, Amsterdam (first ed., North-Holland, Amsterdam, 1993).

Nemat-Nasser, S., Li, J.Y., 1999. Electromechanical response of ionic polymer-metal composites, J. Appl. Phys., to appear.

Oguro, K., Asaka, K., Takenaka, H., 1993. Polymer film actuator driven by low voltage. In: Proceedings of the Fourth International Symposium on Micro Machine and Human Science, Nagoya, pp. 39-40.

Shahinpoor, M., 1992. Conceptual design, kinematics and dynamics of swimming robotic structures using ioinc polymeric gel muscles. Smart Mater. Struct. 1, 91-94.

Taya, M., Arsenault, R.J., 1989. Metal Matrix Composites, Thermomechanical Behavior. Pergamon, New York.

Xue, T., Trent, J.S., Osseo-Asare, K., 1989. Characterization of Nafion membranes by transmission electron microscopy. J. Membr. Sci. 45, 261-271. 